\title{
Tecnologías de gobierno en la formación de profesionales de la salud en una universidad tradicional
}

Jimena Carrasco-Madariaga'

Marcela Apablaza-Santis'

\section{Resumen}

A partir de la década de 1980 el sistema de educación superior chileno ha experimentado sucesivas transformaciones, orientadas por concepciones de lo social y de los sujetos. Éstas han eludido el debate ciudadano presentándose como el resultado de decisiones técnicas que competen sólo a expertos. En el presente trabajo nos proponemos analizar dichos procesos de reformas en la formación de profesionales de una carrera de la salud en Chile desde un enfoque crítico, a fin de promover mayor espacio para el debate. Se utiliza el marco de gubernamentalidad para guiar el estudio de un caso, de una carrera del área de la salud en una universidad tradicional chilena. Así se analizan las lógicas y las tecnologías de gobierno en diferentes niveles, tales como los marcos legales de las reformas, los lineamientos de la universidad, los instrumentos de control sobre el quehacer docente, entre otros. Se da cuenta de cómo las diferentes tecnologías han ido transformando la profesión académica y la formación profesional, de manera coherente con una mentalidad de gobierno neoliberal. Se concluye que los nuevos modelos de educación superior están transformando los procesos de subjetivación de académicos y estudiantes con base en una marcada lógica de mercado, sin que se promueva la discusión respecto del modelo de sujeto y de sociedad que las reformas promueven.

\section{Palabras clave}

Tecnologías de gobierno - Gubernamentalidad - Educación superior - Chile - Neoliberalismo.

I- Universidad Austral de Chile, Valdivia, Chile.

Contactos: jimenacarrasco@uach.cl, marcela.apablaza@uach.cl 


\title{
Government technologies in health professionals' training in a traditional university
}

Jimena Carrasco-Madariaga'

Marcela Apablaza-Santis'

\begin{abstract}
Since the 1980s, the Chilean higher education system has undergone successive transformations oriented by conceptions about the social issue and subjects. These transformations have avoided citizens' debate by being presented as the result of technical decisions that are the concern of experts only. In this paper, we propose to analyze these processes of reform in relation to the training of professionals of a health career in Chile from a critical approach, to promote greater room for debate. We have used the governmentality framework to guide a case study about a health career in a traditional Chilean University. Government logics and technologies are analyzed at different levels, such as the legal frameworks of the reforms, the guidelines of the university, the instruments of control over the professors' work, among others. The results expose how different technologies have transformed the academic profession and professional training, coherently with a neoliberal government mentality. It is concluded that, based on a strong market logic, the new models of higher education are transforming the processes of subjectivation of scholars and students, without promoting the discussion about the model of subject and society that these reforms foster.
\end{abstract}

\section{Keywords}

Government technologies - Governmentality - Higher education Chile - Neoliberalism.

I- Universidad Austral de Chile, Valdivia,

Chile.

Contacts: jimenacarrasco@uach.cl,

marcela.apablaza@uach.cl 


\section{Introducción}

El sistema de educación superior en Chile y en el mundo ha sufrido una serie de trasformaciones. Éstas se presentan como algo necesario en pos de los cambios en el contexto actual, haciendo alusión a una opción por una economía basada en el conocimiento, propia de las reformas en el Espacio Europeo de Educación (CARAMÉS, 1999).

Las razones por las cuales las autoridades han tomado esta opción y no otra, al igual que ocurre en otras decisiones políticas, son presentadas siempre con base en criterios técnicos, con lo cual su validez se da por sentada. De esta forma la opción se transforma en la única posible.

Sin embargo, la educación, así como muchos otros ámbitos que involucran a personas y grupos, no puede reducirse exclusivamente a aspectos técnicos, ya que involucra procesos de subjetivación, sobre los que se establecen una serie de estrategias para que los sujetos incorporen determinadas cualidades a fin de hacer de ellos lo que la sociedad espera o necesita. Dichas estrategias se sostienen sobre ciertas ideas acerca de lo que es un sujeto, que a su vez se relacionan con determinadas posturas ideológicas, que contribuyen a producir un determinado tipo de individuo, es decir, tienen un efecto performativo sobre la subjetividad misma (BLANCO; TEJERINA, 2005; GERGEN, 2001; GONZÁLEZ PARDO; PÉREZ ÁLVAREZ, 2007; LEAL FERREIRA, 2001; PARKER, 2010).

Bajo estos supuestos, entenderemos las transformaciones en los sistemas de educación superior como mutaciones en las estrategias de subjetivación, que responden a cambios en las ideas sobre el tipo de sujeto que debe resultar, y estos a su vez, como producto de constantes interacciones entre distintos actores y agentes existentes en la arena política, económica y social. En estas interacciones se dan relaciones de poder que coaccionan y generan mecanismos de control para alcanzar sus objetivos, mediante la cristalización en reformas de políticas públicas (ACOSTA, 2002). Más aún, proponemos que estas obedecen a cierta racionalidad de gobierno, por lo que no pueden ser analizadas ni comprendidas sino como el resultado de fuerzas que son producto de relaciones de poder, que se materializan en dispositivos que conducen a los agentes hacia comportamientos legitimados y establecidos como lo normativo, generando así procesos de subjetivación en los individuos (COMMISSO, 2013; FOUCAULT, 2006; LEMKE, 2011; YOUDELL, 2006).

En específico, en lo referente a la formación profesional en salud, en Chile así como en muchos países occidentales, se han adoptado las directrices del Espacio Económico Europeo, sin una mayor discusión. En esta línea, se ha incorporado el modelo de Formación Basada en Competencias, como la única posibilidad ante el actual escenario mundial (LAFUENTE et al., 2007). Al mismo tiempo, desde diferentes ámbitos se han desarrollado una serie de investigaciones denominadas críticas, que apuntan a cuestionar los supuestos sobre los cuales se han asentado los procesos de reformas en dicho escenario (ENGEBRETSEN; HEGGEN; EILERTSEN, 2012; FAIRCLOUGH, 2008; NARCIANDI; CARLOS; LEAL FERREIRA, 2011; PINI, 2010). Este tipo de aproximaciones, sin embargo, son poco frecuentes en el ámbito de la formación en salud en el contexto chileno. Por tal motivo consideramos necesario llevar a cabo investigaciones en el campo de la formación de los profesionales de la salud en Chile, que apunten a una comprensión crítica de los procesos de reforma.

Nuestra intención no es denunciar las relaciones de poder presentes en los dispositivos de educación superior, porque creemos que no es posible una educación libre o independiente del ejercicio del poder. Tampoco se trata de rebatir los argumentos técnicos que se han dado para justificar las reformas, ni menos aún hacer un llamado a su sustitución por otros más adecuados. Eso sería una mirada reproductora, es decir, aceptar que hay conocimientos capaces de respaldar por sí mismos las políticas 
educativas, independiente de las condiciones sociales, políticas o económicas.

En concreto, nos proponemos plantear que las actuales transformaciones en la educación superior en Chile, generan prácticas de subjetivación tanto de docentes como de los futuros profesionales, caracterizadas por rasgos que responden a una determinada lógica política. Finalmente, lo que nos interesa es develar cómo el asunto de la educación superior en salud no responde sólo a cuestiones técnicas y proponer que, éstas son en última instancia políticas, y por ende, se deben abrir al debate respecto de sus efectos y cómo estos se relacionan con el tipo de sujeto y de sociedad que deseamos.

Para cumplir con dicho propósito, inicialmente introduciremos algunos elementos conceptuales que ayuden a comprender la relación entre la política y los procesos de subjetivación. En este sentido, haremos una breve reseña de la perspectiva de gubernamentalidad con el propósito de otorgar un marco para comprender las formas en que se ejerce el gobierno y llevar la atención hacia las prácticas y tecnologías menos evidentes del poder para así transitar a un análisis crítico en la revisión de las políticas vigentes (MCKEE, 2009).

Luego, a través de un estudio de caso, analizaremos cómo las actuales transformaciones en la educación superior en Chile, desde una perspectiva de gubernamentalidad, se pueden entender como técnicas y estrategias que buscan alinear a los sujetos con los fines de gobierno. Por medio de este ejercicio analizaremos las diferentes tecnologías de gobierno aplicadas en un programa de formación universitaria del área de la salud, en una universidad tradicional. Se pretende dar cuenta de cómo se logra que los sujetos participen de ciertos fines coherentes con una lógica de gobierno neoliberal.

\section{El marco de la gubernamentalidad}

Gubernamentalidad es un concepto desarrollado por Foucault (2006) para dar cuenta de la mentalidad con que se gobierna a las personas en los estados modernos. Esta noción ha dado bases para un marco de referencia que permite comprender el ejercicio del poder en la actualidad. Su desarrollo ha servido para mostrar cómo el Estado soberano moderno y el individuo autónomo moderno están estrechamente relacionados de manera que cada uno es central en la aparición del otro (FOUCAULT, 1991). Esta idea implica renunciar a una concepción de poder, como algo que se puede localizar en un determinado grupo, ideología o en el Estado. A cambio de esto Foucault se centra en la microfísica del poder.

Desde las nociones de saber poder, Foucault (1990) nos propone que el sujeto moderno es constituido como un objeto de conocimiento y así es también hecho un objeto de control, entendiendo que a la base de todo lo que la ciencia busca conocer, hay un ejercicio de poder sobre un objeto que se busca controlar. Por otra parte, con las tecnologías del Yo, Foucault (1988) nos propone que en la actualidad los individuos han llegado a verse a ellos mismos como objetos de conocimiento y de control, y así cada sujeto ha podido desarrollar una relación consigo mismo y técnicas para lograr el auto control (ROSE, 1996).

La noción de gubernamentalidad, según De Marinis (1999) contiene dos elementos: las racionalidades politicas y las tecnologias de gobierno. Las primeras tienen forma de moral y se elaboran en un campo del deber ser como ideales y principios desde los cuales el gobierno debería estar dirigido (libertad, justicia, igualdad, responsabilidad, ciudadanía, etc.). Las segundas, son los elementos que se utilizan para hacer efectivo y perfectible el ejercicio del poder. Ambas se encuentran unidas en la práctica por una misma racionalidad, de manera que no es posible estudiar las tecnologías de poder sin un análisis de la racionalidad política en la que se basan.

Foucault propone que para comprender las experticias y técnicas que operan en la actualidad, es necesario pensarlas desde una perspectiva de racionalidad de gobierno, es decir, no como 
una institución, una ideología, una teoría o una filosofía de la libertad individual, sino como una forma de hacer pensable y practicable una cierta actividad de gobierno. La gubernamentalidad, hace referencia a esto: implica una cierta mentalidad o pensamiento que es internalizada por los individuos y los guías (FOUCAULT, 1991), de manera de hacer posible el auto gobierno, es decir, una forma de conducción en la que el individuo juega un rol activo.

Rose (1997) sugiere que, desde esta perspectiva, es posible entender cómo las múltiples tácticas de gobierno son reunidas en un sólo pensamiento, lo que les otorga una coherencia lógica, posibilitada actualmente por el liberalismo, a ojos de este autor. Argumenta que el sujeto libre sólo es posible en cierta condición social y cultural, que corresponde a la del individuo que participa libremente de los fines de gobierno, sin que sea necesario obligarlo. En este contexto la libertad no es algo opuesto al gobierno, sino un recurso clave, pues a través del alineamiento entre las libertades individuales y las estrategias liberales de gobierno, se direcciona la libre elección de las personas, motivándolos a trabajar por los fines de gobierno. "El sujeto así creado podría producir los fines de gobierno por el cumplimiento de los mismo, más que por ser sólo obediente, por ser libre de una forma específica” (ROSE; O'MALLEY; VALVERDE, 2006, p. 89).

Esto no se debe entender como una falsa libertad, si asumimos que, como propone Foucault, no se puede pensar el poder sin la resistencia. Esto quiere decir que el conocimiento y las tecnologías producidas no se imponen a los sujetos como reglas de comportamiento, sino que son incorporados y apropiados por éstos en diferentes formas. Proceso que ocurre al mismo tiempo en que éstos van introduciendo transformaciones, incluso a partir de formas de resistencia a las lógicas de gobierno.

La introducción de tecnologías o técnicas del yo implica una mayor conexión entre la subjetivación y sujeción (BURSCHELL, 1996), es decir, entre el comportamiento que el sujeto tiene en su vida diaria y el mandato moral o las reglas que norman una sociedad. El individuo ahora tiene la oportunidad de influir en la manera en que se construye su subjetividad al hacer de sí mismo un objeto de conocimiento y de control; puede interpretar las normas de comportamiento y decidir en relación a ellas en vez de sólo cumplirlas.

Las racionalidades políticas, es decir, los ideales de gobierno, no se deben comprender como aspectos que se imponen, sino como elementos que pueden ser incorporados por los sujetos de forma voluntaria en sus procesos de auto construcción. Cuando esto ocurre, podemos decir que hay un punto de contacto entre las técnicas de dominación y las técnicas del yo que producen posiciones de sujeto (BURSCHELL, 1996). De hecho, para que los discursos de racionalidad política funcionen, necesitan que los sujetos se identifiquen con ellos, es decir, que sean vistos como compatibles con sus intereses. Así se entiende que el poder es algo siempre en juego: el sujeto actual, entendido como una constitución discursiva, es al mismo tiempo compatible con, y resistente a las lógicas de gobierno (WEEDON, 1987).

En suma, las tecnologías de gobierno son mecanismos, estrategias y procedimientos, que conectan las aspiraciones de las autoridades y las actividades de los individuos, estructurando $\mathrm{y}$ dando forma a las posibles acciones de los sujetos, de manera de orientar sus elecciones (sin obligarlos), a fin de producir ciertos efectos.

\section{Estrategias de investigación}

Se realizó un diseño de corte exploratorio, cualitativo y crítico tendiente al análisis de los discursos dominantes en los procesos de reforma de una carrera de la Salud en una universidad chilena tradicional. Para la organización de los resultados se buscó detectar los elementos clave en los procesos de subjetivación. Dado que no se pretende sacar a la luz algo difícil de percibir, no se recurrió a una forma específica de método de análisis crítico del discurso, sino más bien a 
la clasificación de elementos que consideramos que nos permiten articular una comprensión crítica del fenómeno.

Realizamos una revisión de la literatura vinculada a los procesos de reforma de los sistemas de educación superior en Chile, de acuerdo al modelo triangular propuesto por Clark (1983 apud BRUNNER, 2009). Luego se revisaron documentos relacionados al actual periodo de transformaciones de la política educacional, a nivel nacional e internacional. Los principales documentos internacionales analizados fueron aquellos relacionados a directrices en educación superior de 1) la Comunidad Económica Europea 2) Banco Mundial y 3) Organización para Cooperación y Desarrollo Económico (OCDE). A nivel nacional se analizaron 1) el Programa de Mejoramiento de la Calidad de Educación Superior (MECESUP) 2) la Ley 20.129 sobre Sistema Nacional de Aseguramiento de la Calidad de la Educación Superior y 3) las normativas que regulan el funcionamiento de la Comisión Nacional de Acreditación de Pregrado (CNAP).

Luego delimitamos el caso de estudio de acuerdo a diferentes niveles en la organización de la institución de educación superior para comprender cómo las políticas impactan en la organización de la institución en los diferentes niveles. Para esto se analizaron documentos posteriores a la entrada en vigencia de la Ley 20.129 sobre Sistema Nacional de Aseguramiento de la Calidad de la Educación Superior, y que otorgan directrices para la formación de pregrado y el desempeño de los académicos. Estos fueron: 1) Plan Estratégico y 2) Modelo Educativo de la Institución. Por último, se analizaron documentos que guiaron el proceso de auto evaluación de una carrera del área de la salud, tales como guías emanadas de la Comisión Nacional de Acreditación, y documentos de la Oficina de Auto Evaluación Institucional de la casa de estudios.

Las principales categorías para el análisis fueron: 1) procesos de neoliberalización de la educación chilena y 2) procesos de subjetivación de académicos y de estudiantes. Del cruce de ambas, se desprenden diferentes formas de articulación de las tecnologías de gobierno con los mecanismos de control implementados por la institución de educación superior y los procesos de subjetivación de académicos y estudiantes.

\section{Resultados}

\section{Transformaciones en el sistema de educación superior chileno desde una perspectiva de gubernamentalidad}

El modelo triangular propuesto por Clark (1983 apud BRUNNER, 2009), nos permite comprender cómo las universidades han variado su coordinación y han establecido nuevos sistemas de orden en respuesta a la racionalidad cambiante. En esta propuesta teórico-analítica, el autor distingue tres sistemas clave; el sistema estatal, el sistema de mercado y el sistema profesional o académico, cuya interacción evidencia los desplazamientos del eje predominante y las consecuencias y transformaciones que traen consigo en el comportamiento y organización de las instituciones de educación terciaria (BRUNNER, 2008, 2009).

Bajo este modelo, en el caso de Chile, dicha transformación puede ser resumida en cuatro periodos principales (BRUNNER, 2009). En primer lugar, el periodo previo al Golpe de Estado de 1973, con un predominante eje de poder centrado en las oligarquías académicas, donde las instituciones universitarias gozaban de plena autonomía, y cuya relación con el Estado era definida como benevolente, limitada a la provisión de recursos sin ningún tipo de control sobre la calidad de la enseñanza, el gasto y su organización interna.

En segundo lugar, el periodo entre 1973 y 1980 que se inicia con la dictadura, se caracteriza por un desplazamiento de dicho eje hacia el control del Estado, coherente con estrategias autoritarias, con el fin de sanear las universidades, de elementos amenazantes de filiación marxista (BRUNNER, 2009). En este periodo, las instituciones mantienen 
la provisión de recursos incondicional por parte del Estado, pero son despojadas de todo tipo de participación en las decisiones de su organización interna, exceptuando algunas menores como la definición de la cantidad de estudiantes que ingresan.

Posteriormente, con la reforma constitucional de 1981 y su posterior materialización en la Ley Orgánica Constitucional de Enseñanza (LOCE) de 1989, Chile se abre a un nuevo sistema educacional, en el que el eje predominante se desplaza hacia el mercado. Este cambio se basa en el principio de la libertad de enseñanza, lo que sumado a un estrechamiento del rol regulador y financiero por parte del Estado, permite la aparición de nuevos proveedores privados. La competencia entre las instituciones, pasa a ser el principal regulador del crecimiento y de la calidad de la enseñanza superior (BRUNNER, 2008, 2009; CORNEJO, 2013). Dentro de las principales reformas, se destaca el fin del sistema de financiamiento gratuito hacia los estudiantes y sus familias, en cuyo reemplazo se implementa el nuevo esquema de financiamiento compartido, basado en el cobro de aranceles, disminución del Aporte Fiscal Directo (AFD) (asignación estatal donde el 95\% del subsidio es destinado directamente y de libre gasto. El 5\% restante, según desempeño anual de las instituciones en base a indicadores de matrícula, cantidad de académicos con posgrados, proyectos de investigación y publicaciones de alta calidad) (CHILE, 2015) e introducción del Aporte Fiscal Indirecto (AFI) (Asignación estatal entregada a todas las instituciones de educación superior que incorporen en sus matrículas al 20\% de los estudiantes con mejores puntajes en la prueba de selección universitaria correspondiente a su primer año de estudios académico) (CHILE, 2015) y el crédito universitario; y la diversificación de la educación terciaria (universidades, institutos profesionales y centros de Formación Técnica) (BRUNNER, 2009; OCDE, 2009).

Finalmente, un cuarto periodo, se inicia con el retorno de la democracia a partir de
1990. Los gobiernos reconocen las políticas del periodo previo como exitosas en cuanto a acceso, pero se proponen avanzar hacia la calidad. Se buscará seguir avanzado en la expansión de la matrícula, y comienza una mayor preocupación en materia de eficiencia del sistema (ACOSTA, 2002; BRUNNER, 2009). El mayor cambio experimentado en este periodo es la devolución de la autonomía a las universidades, sin comprometer con esto, el sistema de financiamiento mixto y la libertad de enseñanza, con lo que el mercado permanece como preponderante en dicha interacción.

En sintesis, bajo un escenario de fuerte globalización y neoliberalización, el sistema de educación superior chileno actual se puede comprender como resultado de un proceso de transformación dado en la interacción entre las universidades, el Estado, y el mercado, donde este último se posiciona como un agente dominante (ACOSTA, 2002; BRUNNER, 2008, 2009, 2011; COMMISSO, 2013; CORNEJO, 2013), cuyos principios fundamentales son la eficiencia, la efectividad y la nueva administración pública (ACOSTA, 2002; BRUNNER, 2009).

Luego, aplicando la noción de gubernamentalidad, comprendemos que los principios que guían estas transformaciones, reflejan una racionalidad de mercado (COMMISSO, 2013) que es también una racionalidad de gobierno. Así, la lógica de mercado es coherente con estrategias de gobierno, con lo cual, contrario a lo que se cree, se genera un mayor control desde el Estado a través de tecnologías y dispositivos que consolidan un sistema basado en lo que se conoce hoy como cuasimercardo (BRUNNER, 2009; MORDOCHOWICZ, 2008; SANTIAGO; CARVALHO, 2012): El Estado promueve un comportamiento de competitividad mediante el uso y despliegue de tecnologías de control.

Así los principios que guían estas transformaciones deben ser coherentes con una racionalidad de mercado (COMMISSO, 2013), no sólo en lo referente a las formas de administración, sino también en torno al objeto 
de gobierno. El régimen de conocimiento/ aprendizaje como foco de gobierno, ya no se entiende como una entrega de conocimiento desde una oligarquía académica, ni como un derecho que debe ser garantizado por el Estado sin condicionar su resultado a los procesos de globalización del capital; el conocimiento/ aprendizaje ahora se comprenderá como constituido por las lógicas del mercado: como el resultado de interacciones entre diferentes actores, donde la lógica que predomina y media las interacciones, es la de competitividad.

\section{Tecnologías de gobierno y subjetivación académica}

Un contexto de mercados educacionales establece un sistema de fuerzas sobre los otros agentes, lo cual provoca que tanto el Estado como las universidades, desplieguen una serie de estrategias, destinadas a regular a distancia dichas instituciones y a enfrentar las demandas del mercado por medio del autocontrol o autogobierno de sus agentes (COMMISSO, 2013; GORNITZKA, 1999; SUSPITSYNA, 2010). Esta nueva racionalidad, es vista como un conjunto de políticas de carácter performativo, es decir, constitutivas de los sujetos, cuyo poder de impacto recae en la conformación de procesos de subjetivación de los mismos individuos (YOUDELL, 2006).

En el caso en estudio, esto se ve reflejado en la forma en la que la universidad como institución, entiende el contexto al cual se enfrenta, caracterizado por la "alta competitividad en términos de accesos a recursos públicos, lo que obliga a cambiar ciertos paradigmas" (UACh, 2012, p. 20). A partir de este escenario se deben planificar las estrategias de cambio para toda la institución.

La Ley de Sistema de Aseguramiento de la Calidad de la Educación Superior propuso un sistema de financiamiento principalmente a la demanda, es decir, hacia los estudiantes, en base a créditos y becas, bajo la modalidad voucher. Por otra parte, estableció un esquema de financiamiento hacia el producto o desempeño de las instituciones, por medio de fondos de investigación -Aporte Fiscal Indirecto-, convenios de desempeño, fondos por acreditación, entre otros (BRUNNER, 2009), los que serían entregados bajo una serie de tecnologías de accountability, es decir, entrega de recursos condicionada a metas, las que a su vez coaptan a los agentes de las instituciones a modificar sus intereses, prácticas y objetivos, conformando así una nueva subjetividad en los individuos del nivel medio como del nivel micro o intersticial (SUSPITSYNA, 2010).

Con la finalidad de ser competitiva ante las otras instituciones, se han desplegado una serie de dispositivos y tecnologías (ACOSTA, 2002; BAY, 2011; BRUNNER, 2009; COMMISSO, 2013; GORNITZKA, 1999; SANTIAGO; CARVALHO, 2012; SUSPITSYNA, 2010). El cuerpo académico inmerso en esta lógica de mercado, es coaccionado a transformar su conducta, lo que se puede comprender en el marco de una especie de capitalismo académico (BRUNNER, 2009): las instituciones en competencia buscan la captación de aquellos académicos de mayor prestigio, y éstos, a su vez, la transacción de sus productos a cambio de recursos de financiamiento, ya sea por medio de incentivos económicos internos, adjudicación de fondos de investigación, desarrollo de productos bajo propiedad intelectual como es el caso de patentes y publicaciones, o de un estatus para acceder a mejores alternativas laborales, ya sea de manera interna (promoción académica) o en otra institución de mayor prestigio y/o sueldos más altos, por lo que la profesión docente ha debido internalizar dichas estrategias y en consecuencia, modificar no sólo sus prácticas, sino también su racionalidad respecto del ejercicio académico. En esto juegan un rol central las tecnologías como el accountability, que pasan a formar parte esencial de las prácticas de la nueva profesión académica.

En respuesta a esta racionalidad, nominada por algunos exponentes como el sistema de managerialismo (BAY, 2011; BRUNNER, 2009), las universidades deben 
transformar su cultura organizacional, por medio de diversas tecnologías que penetran en la cotidianeidad de sus agentes, llegando incluso a transformar su identidad profesionalacadémica (COMMISSO, 2013).

La racionalidad antes centrada en un régimen de conocimiento/aprendizaje desarrollado de forma autónoma y de plena orientación hacia la actividad académica, ahora es reemplazada por el managerialismo, que surge como una alternativa de supervivencia y adaptación a las nuevas demandas que deben enfrentar las universidades chilenas.

Uno de los resultados de esta nueva forma de administración, es la modificación en la relación entre los integrantes del cuerpo académico. Con el fin de la dictadura, si bien se amplían las libertades de participación de éstos, no se retorna al pasado. Se debe implementar un sistema democrático moderno, jerárquico neoliberal, conformado por juntas directivas en las que la oligarquía académica como autoridad, es reemplazada por expertos en las nuevas formas de administración y los académicos son relegados a sujetos cuyas funciones se limitan a producir conocimiento-aprendizaje.

En el caso en estudio, una de las aspiraciones centrales de la actual rectoría, ha sido generar instrumentos de planificación para una programación de las actividades académicas, administrativas y financieras. Para esto, habría sido necesario constituir un equipo de trabajo, que elaboró el Plan Estratégico 2012-2015:

[...] el cual incorporó por primera vez los Convenios de Desempeño como herramienta de gestión, estructurado sobre la metodología de Balanced Scorecard y monitoreado a través del Tablero de Comando. (UACh, 2012, p. 5).

Este cambio en el estamento académico, involucra una serie de transformaciones en la normativa interna, que coaccionadas por las políticas externas, en su conjunto configuran un sistema diferente constitutivo de una nueva docencia, una nueva forma de investigación y una nueva necesidad de vinculación con la comunidad, articuladas bajo una lógica de producción e intercambio. En el caso en estudio, esto se evidencia en la definición que se hace en el plan estratégico actualmente vigente, de cinco áreas fundamentales, que además de la docencia, investigación y vinculación con el entorno, incorpora Gestión y Desarrollo y Reformas Estructurales. A la vez, se dice que la Universidad ha logrado una cultura organizacional planificada, y se hace un llamado explícito a "cada uno de los integrantes de la comunidad universitaria, a generar una cultura de la planificación y “accountability” (p. 5).

En estas nuevas formas de subjetivación del sujeto docente, juegan un rol determinante tecnologías de gobierno centradas en el accountability: prácticas basadas en la transparencia, como el registro de cada una de las acciones desempeñadas por sus agentes, (BAY, 2011; GORNITZKA, 1999; SUSPITSYNA, 2010), pago mediante incentivos o compromisos de desempeño por producción (publicaciones) y sistemas de promoción o jerarquización. Luego, en el caso en estudio, dentro de las líneas estratégicas, se incorpora un programa de "Modernización e Integración de los sistemas de Información y Gestión” (UACh, 2012, p. 36) que tiende a perfeccionar los sistemas ya existentes para el registro e información de las actividades de las/los docentes y el uso de esta información en la gestión. Los académicos deben tender a transformar sus prácticas orientándolas a producción contra tiempo, $\mathrm{y}$ haciendo prevalecer los intereses a los que debe responder la institución de acuerdo a los vaivenes del mercado (GORNITZKA, 1999; SUSPITSYNA, 2010).

\section{La formación por competencias como formas de subjetivación en los procesos de enseñanza aprendizaje en salud}

En el año 1997 se crea el Programa de Mejoramiento de la Calidad de Educación 
Superior (MECESUP), a partir del cual se busca que las universidades chilenas rediseñen sus planes de estudio para convertirlos en currículos basados en el modelo de competencias. Este programa se implementó como un fondo concursable al que las universidades podían acceder para la dotación de recursos físicos y humanos, a cambio de un proceso de rediseño curricular basado en enfoque por competencias. Luego, en el año 1999, se crea la Comisión Nacional de Acreditación de Pregrado (CNAP) cuyo objetivo es el aseguramiento de la calidad. Dicho organismo será el encargado de efectuar procesos de acreditación institucional y de carreras. Esto es formalizado con la promulgación de la Ley 20.129 sobre Sistema Nacional de Aseguramiento de la Calidad de la Educación Superior que regula el licenciamiento de instituciones, acreditación institucional y de programas de formación a través de la creación de la Comisión Nacional de Acreditación, el 23 de octubre de 2006.

Entre los requisitos de acreditación se establece que, las carreras deben contar con un perfil de egreso, consistente con la estructura curricular, el plan de estudios y los métodos pedagógicos. En esta línea, la estructuración del currículo se elabora en función del perfil de egreso considerando las competencias profesionales y desempeños de carácter general (CHILE, 2008). Alineándose con esto, la universidad en estudio, por medio del decreto $n^{\circ} 193$ del año 1999 crea la Oficina de Auto Evaluación Institucional, la que tendrá dentro de sus funciones:

Planificar y organizar el proceso global de autoevaluación académica al interior de la Universidad, de acuerdo a los lineamientos y políticas contenidas en el Proyecto MECESUP del Ministerio de Educación" y "Coordinar y asesorar a los grupos de trabajo que se organicen en cada una de las Facultades para llevar adelante los procesos de autoevaluación y acreditación académica. (UACh, 2005, p. 2).
Luego en otro decreto del año 2005 establece la adscripción al modelo curricular por competencias.

El modelo por competencias en Chile, ha sido analizado desde perspectivas críticas (ESPINOZA, 2012). Aquí se problematiza "Como diría Foucault, cómo es que hablamos de esto y no de otra cosa, cómo es que este discurso foráneo a la educación universitaria encuentra su orden legítimo en ella, y se posiciona con tal fuerza performativa" (p. 183). De un ejercicio genealógico, se concluye que la emergencia del discurso de las competencias se puede explicar por la mercantilización de la educación superior, y más especificamente por la tendencia a que las universidades se adecuen a las necesidades del mercado laboral y de la expansión del mercado de la educación superior (ESPINOZA, 2012; FERNÁNDEZ, 2009). Surge fuera de la academia, en el ámbito de la formación técnica y de teorías conductistas del aprendizaje. Sus premisas se pueden rastrear en las ideas de Taylor (1913 apud ESPINOZA, 2012) sobre la necesidad de separar la planificación del trabajo y su ejecución, donde el desempeño esperable de un determinado profesional (trabajador) es definido por un grupo de expertos, cuyo conocimiento se impone a lo que puedan decir los propios profesionales/trabajadores, e incluso los encargados de su formación (los académicos).

La formación por competencias en educación superior emerge en el marco de la Comunidad Económica Europea y busca hacer comparables los grados y títulos a fin de facilitar la circulación de los estudiantes y profesionales por el espacio europeo (ESPACIO..., 1999). Así, la compatibilidad internacional de las certificaciones se volverá relevante para la circulación en un mercado competitivo. Posteriormente, desde organismos internacionales se plantea la necesidad de que los países desarrollen su capital humano, con el objeto de generar competitividad laboral. Cobra relevancia la internacionalización de la enseñanza, como una estrategia de regular 
y normativizar los estándares de calidad esperados (OCDE; BANCO MUNDIAL, 2009).

Bajo esta lógica, la educación se asimila a un proceso de producción, que debe establecer claramente lo que produce y su resultado debe además ser fácilmente transferible $\mathrm{y}$ acumulable, como un bien que se pueda transar en el mercado. Para eso es necesario un medio de transacción, algo que permita comparar y hacer equivalentes los productos del proceso de la educación, independiente de sus diferentes orientaciones y orígenes. De ahí la insistencia en la necesidad de los créditos transferibles -un sistema de transferencia y acumulación de los resultados del proceso de educación (EUROPEAN COMMUNITIES, 2004), es decir, una moneda de cambio (GONZÁLEZ; WAGENAAR, 2003).

Como estrategia de legitimación, el discurso de la formación por competencia se ha presentado como el resultado del acuerdo de todos los actores involucrados en la búsqueda de una solución urgente a la actual situación de la educación superior, que es resultado de la globalización de la economía, y que hace necesario el desarrollo de un capital humano capaz de generar competitividad laboral ante los otros países. Es decir, la necesidad de un cambio toma enunciados que tienen su origen en los análisis económicos, que luego transitan al ámbito educativo y se instalan como verdades incuestionables a las que los sistemas educativos deben responder (ESPINOZA, 2012). Estos procesos se presentan como hechos, es decir, como realidades neutrales y objetivas, que se pueden analizar desde la racionalidad propia de un positivismo científico, a fin de generar el conocimiento necesario para su abordaje. Este tipo de acercamiento, sin embargo, no da cuenta de las relaciones de poder en las que se fraguan estos hechos y conocimientos. Por tanto, desde la perspectiva de gubernamentalidad, cabe preguntarse sobre las tecnologías de gobierno y tecnologías del yo que se ponen en contacto en estas nuevas formas de subjetivación/educación.
El proceso de enseñanza/aprendizaje basado en competencias, debe integrar diferentes aspectos tales como conductas, comportamientos, valores y actitudes (GONZÁLEZ; WAGENAAR, 2003) y no limitarse sólo a la adquisición de conocimientos. Para esto, deben considerarse aspectos de la subjetividad de los estudiantes: nivel social y económico, cultura, raza, género, etc. En el caso en estudio, se elaboró un informe del perfil psicoeducativo de los estudiantes que ingresan a la carrera, como una herramienta destinada a orientar las acciones en el proceso de enseñanza/aprendizaje (UACh, 2013). Aspectos como autoestima, formas de procesar la información, lugar de procedencia, nivel social y cultural configuran dicho perfil y deben ser las variantes a considerar para lograr las competencias de egreso.

Podemos decir entonces, que las nuevas orientaciones en educación superior deben incorporar la subjetividad del estudiante: no basta con que éste adquiera conocimientos, sino que debe además asumir ciertas formas de ser, incorporando de forma planificada aspectos personales y morales (CASARES; CARMONA; MARTÍNEZ-RODRÍGUEZ, 2010). Se dice que el proceso debe estar centrado en las características del estudiante, es decir, en aspectos subjetivos como los mencionados anteriormente. Sin embargo, el perfil de egreso define cómo debe llegar a ser este sujeto/ estudiante con base en las necesidades del mercado laboral y se establecen los mecanismos para lograr que los sujetos/estudiantes lleguen a ser de esa forma (GONZÁLEZ; WAGENAAR, 2003; KENNEDY, 2007). Las características que son deseables son aquellas compatibles con el mercado laboral y su lógica competitiva, lo que se traduce en el predominio de la dimensión técnica del quehacer profesional por sobre las dimensiones sociales, políticas, culturales y éticas (ESPINOZA, 2012). En el caso analizado, siguiendo los lineamientos de la Comisión Nacional de Acreditación y de la Oficina de Evaluación Institucional, se realizó un proceso 
de validación del perfil de egreso que consistió en una consulta a empleadores sobre las características que consideraban deseables en estos profesionales para su contratación.

La necesidad de un cambio en las metodologías de enseñanza y aprendizaje, hacia un enfoque centrado en el estudiante, ha sido uno de los principales puntos de anclaje para la introducción de la formación por competencias en los sistemas de educación chilena (ESPINOZA, 2012) y ha encontrado eco en los académicos de la Facultad del caso en estudio (JABIF, 2007; MÁRQUEZ, 2012).

La formación centrada en el estudiante supone que el docente desparece como ente de control externo. Los principios y valores que deberán guiar las acciones de las/los estudiantes se entienden como aspectos que deberán ser internalizados, para la adquisición auténtica de los aprendizajes. Sin embargo, desde una perspectiva de gubernamentalidad podemos entender que los valores a ser internalizados son coherentes con la lógica de gobierno neoliberal y que para que desaparezca la figura del docente (control externo), debe articularse una serie de tecnologías de gobierno con tecnologías del Yo. De ahí la importancia de conocer los aspectos de las subjetividades de los individuos.

En el caso analizado, el proyecto inicial de la carrera fue formulado con el marco de formación por competencias y desde su apertura en el año 2004, los programas de estudio de las asignaturas dictadas por el equipo de docentes del área disciplinar, han sido elaborados bajo este marco. A lo largo del tiempo, este equipo docente ha ido incorporando las diferentes orientaciones del Departamento de Aseguramiento de la Calidad e Innovación Curricular (DACIC) de la Universidad, dentro de las cuales destaca la elaboración de un perfil de egreso en el año 2010, que se estructura en competencias. Actualmente, en el marco del proceso de auto evaluación de la carrera, se ha debido actualizar el proyecto original, incorporando el Modelo Educativo de la Institución (JABIF,
2007; UACh, 2007) el cual adscribe al modelo de formación por competencias y establece ejes y ciclos de formación.

En este proceso se han debido implementar nuevas metodologías de enseñanza/aprendizaje: se han aumentado progresivamente las tutorias en pequeños grupos, se han debido formular y aplicar pautas de evaluación de desempeños esperados, que incorporen aspectos como actitudes y valores de las/los estudiantes, implementación de estrategias remediales, es decir, orientadas a no retrasar el avance curricular de las/los estudiantes, asegurando el logro de las competencias establecidas en los programas. Todas estas acciones han significado un aumento de la carga en docencia de pre grado, además de un proceso paulatino de incorporación de los términos del marco de Formación por Competencias. Esto se ve reflejado en el lenguaje y en las prácticas de las/ los docentes, que a su vez, modifican el lenguaje y las prácticas de las/los estudiantes, dando cuenta de nuevos procesos de subjetivación, que reflejan el alineamiento de los sujetos con determinados fines de gobierno.

\section{Conclusiones}

El proceso de mercadización, provoca un cambio en la relación entre Estado e instituciones, donde el mercado se posiciona como un agente dominante. Esto se ve reflejado en regulaciones gubernamentales, en especial las que tienen relación con el financiamiento, que condicionan el aporte de recursos desde el Estado, y llevan a que las universidades compitan entre ellas (COMMISSO, 2013). La competencia pasa a ser uno de los dispositivos de control más fuertemente instaurados en dichos sistemas, en cuyo efecto las instituciones deben modificar sus comportamientos organizacionales internos, para así adoptar un sistema de administración basado en el managerialismo, el cual se inspira en los principios de la nueva administración pública, enfoque que le permitiría competir por recursos financieros públicos y privados 
(BRUNNER, 2008; 2009; 2011; COMMISSO, 2013; SANTIAGO; CARVALHO, 2012).

Chile avanza en un espiral de mercadización de la educación superior (BRUNNER, 2009) mediante sucesivas reformas educacionales, lo que provoca transformaciones profundas de las tecnologías de control al interior de las universidades y a su vez, de subjetividades de estudiantes, académicos y de la sociedad en su conjunto, enmarcadas éstas en principios neoliberales (CORNEJO, 2013). En este proceso ha cristalizado lo que hoy día se reconoce como un sistema en crisis y centro de convulsión ciudadana y política.

La adecuación de los programas de estudio a un sistema de formación por competencias responde a la misma lógica de mercadización de la educación y resulta por una imposición desde las autoridades de gobierno: los procesos de acreditación universitarias. Luego, las autoridades universitarias deben lograr alinear a toda la comunidad a fin de obtener recursos.

Los procesos de acreditación de las carreras, y la consecuente adecuación a una formación por competencias, han pasado a ser responsabilidad de los académicos, sin cabida a cuestionar la forma ni el fondo de éstos. En el proceso, éstos pueden sentirse identificados y compartir ciertos discursos sobre la necesidad de una formación basada en competencia, pero esto no está ligado necesariamente a un análisis de las causas macro políticas y económicas que justifican estas transformaciones, tales como el Proceso Bolonia y cambios socioeconómicos del país (MÁRQUEZ, 2012). Consideramos que es necesario un análisis crítico de estos procesos desde los académicos y el marco de gubernamentalidad puede ser una herramienta útil en este sentido.

\section{Referencias}

ACOSTA, Adrián. El neointervencionismo estatal en la educación superior en América Latina. Sociológica, México D. F., v. 17, n. 49, p. 43-72, 2002.

BAY, Uschi. Unpacking neo-liberal technologies of government in Australian higher education social work departments. Journal of Social Work, UK, v. 11, n. 2, p. 222-236, 2011.

BLANCO, Florentino; TEJERINA, Jorge Castro. El cultivo de la mente: un ensayo histórico-crítico sobre la cultura psicológica. Revista de Hispanismo Filosófico, Madrid, n. 10, p. 143-146, 2005.

BRUNNER, José Joaquín. Educación superior en Chile: instituciones, mercados y políticas gubernamentales (1967-2007). Santiago de Chile: Universidad Diego Portales, 2009.

BRUNNER, José Joaquín. El sistema de educación superior en chile: un enfoque de economía política comparada. Availação, Campinas, v. 13, n. 2, p. 451-486, 2008.

BRUNNER, José Joaquín. Gobernanza universitaria: tipología, dinámicas y tendencias. Revista de Educación, Madrid, n. 355, p. 137-159, 2011.

BURSCHELL, Graham. Liberal government and techniques of the self. London: UCL Press, 1996.

CARAMÉS, José. La nueva cultura de la Universidad del Siglo XXI: la tercera vía universitaria.[España]: Trabe,1999.

CASARES, Pilar; CARMONA, Gabriel; MARTíNEZ-RODRÍGUEZ, Francisco. Valores profesionales en la formación universitaria. Revista Electrónica de Investigación Educativa, Baja California, n. esp., p. 01-15, 2010.

CHILE. Comisión Nacional de Acreditación (CNA). Manual para el desarrollo de proceso de autoevaluación de carreras de pregrado. Santiago de Chile: CNA, 2008. 
CHILE. Programa de Mejoramiento de la Calidad de Educación Superior (MECESUP). Aporte fiscal directo. Santiago de Chile: Mineduc, 2015.

COMMISSO, Giuliana. Governance and conflict in the university: the mobilization of Italian researchers against neoliberal reform. Journal of Education Policy, UK, v. 28, n. 2, p. 157-177, mzo. 2013.

CORNEJO, Rodrigo. El sujeto docente ausente de las movilizaciones educativas: un análisis del discurso docente. Psicoperspectivas, Valparaíso, v. 12, n. 2, p. 72-82, 2013.

DE MARINIS, Pablo. Gobierno, gubernamentalidad, Foucault y los anglofoucautianos (un ensayo sobre la racionalidad política del neoliberalismo). Madrid: Centro de Investigaciones Sociológicas, 1999.

ENGEBRETSEN, Eivind; HEGGEN, Kristing; EILERTSEN, Heidi. Accreditation and power: a discourse analysis of a new regime of governance in higher education. Scandinavian Journal of Educational Research, Oslo, v. 56, n. 4, p. 401-417, 2012.

ESPACIO EUROPEO DE EDUCACIÓN SUPERIOR. Declaración de Bolonia. Madrid: [s. n.], 1999.

ESPINOZA, Olga. Arqueología del discurso de las competencias en la formación inicial docente en Chile. Santiago de Chile: Pontificia Universidad Católica de Chile, 2012.

EUROPEAN COMMUNITIES. European credit transfer and accomulation system (ECTS): key features. [Bélgica]: Office for Official Publications of the European Communities, 2004.

FAIRCLOUGH, Norman. El análisis crítico del discurso y la mercantilización del discurso público: las universidades. Discurso \& Sociedad, Barcelona, v. 2, n. 1, p. 170-185, 2008.

FERNÁNDEZ, Cesar. ¿Refundar Bolonia? Un análisis político de los discursos sobre el proceso de creación del espacio europeo de educación superior. Revista Española de Educación Comparada, Madrid, n. 15, p. 131-161, 2009.

FOUCAULT, Michel. Governamentality: Hemel Hemstead: Harvester Whaetsheaf, 1991.

FOUCAULT, Michel. Seguridad, territorio, población. Buenos Aires: Fondo de Cultura Económica, 2006.

FOUCAULT, Michel. Techonologies of the self. Amherst: University Massachusetts Press, 1988. A seminar with Michel Foucault at the University of Vermont October 1982.

FOUCAULT, Michel. The history of sexuality: the use of pleasure. New York: Random House Digital, 1990.

GERGEN, Kenneth. Construction in contention toward consequential resolutions. Theory \& Psychology, Ontario, v. 11, n. 3, p. 419-432, 2001.

GONZÁLEZ, Julia; WAGENAAR, Robert. Tunning educational structures in Europe. Deusto: Universidad de Deusto, 2003.

GONZÁLEZ PARDO, Héctor; PÉREZ ÁLVAREZ, Marino. La invención de trastornos mentales: ¿escuchando al fármaco 0 al paciente? Madrid: Alianza, 2007.

GORNITZKA, Áse. Governmental policies and organisational change in higher education. Higher Education, UK, v. 38, n. 1, p. 5-31, jul. 1999.

JABIF, Liliana. La docencia universitaria bajo un enfoque de competencias: orientaciones prácticas para docentes. Valdivia: UACh, 2007.

KENNEDY, Declan. Redactar utilizar resultados de aprendizaje: un manual práctico. Cork: University Collegue Cork, 2007.

LAFUENTE, José-Vicente et al. El diseño curricular por competencias en educación médica: impacto en la formación profesional. Educación Médica, Madrid, v. 10, n. 2, p. 86-92, 2007.

LEAL FERREIRA, Arthur. Por que existem tantas psicologias? Revista do Departamento de Psicologia, Niterói, v. 13, n. 1, p. 9-16, 2001.

LEMKE, Thomas. Foucault, governmentality, and critique. St. Paul: Paradigm, 2011.

MÁRQUEZ, Myriam. El proceso de construcción curricular en el modelo por competencia: estudio de caso del rediseño e implementación del ciclo básico en una carrera de medicina. Santiago de Chile: Pontificia Universidad Católica de Chile, 2012. 
MCKEE, Kim. Post-Foucauldian governmentality: what does it offer critical social policy analysis? Critical Social Policy, UK, v. 29, n. 3, p. 465-486, 2009.

MORDOCHOWICZ, Alejandro. Economía, educación, regulación y mercado: una convivencia difícil. Pro-Posiçoes, Campinas, v. 3 , n. 57, p. 131-157, 2008.

NARCIANDI, Loredo; CARLOS, José; LEAL FERREIRA, Arthur. Aventuras y desventuras de la educación en el Reino de Psicolandia: el supuesto respaldo cientifico del Espacio Europeo de Educación Superior. Athenea Digital, Barcelona, v. 11, n. 3, p. 79-97, 2011.

OCDE - Organización para Cooperación y Desarrollo Económico. Revisión de las políticas nacionales para la educación: la educación superior en Chile. Santiago de Chile: OCDE, 2009.

OCDE - Organización para Cooperación y Desarrollo Económico; Banco Mundial. Revisión políticas nacioanales de educación: la educación superior en Chile. Santiago de Chile: Mineduc, 2009.

PARKER, Ian. La psicología como ideología: contra la disciplina. Madrid: Los Libros de la Catarata, 2010.

PINI, Mónica. Análisis crítico del discurso: políticas educativas en España en el marco de la Unión Europea. Revista de la Asociación de Sociología de la Educación, Valencia, v. 3, n. 1, p. 105-127, 2010.

ROSE, Nikolas. Governing "advanced" liberal democracies. In: ARADHANA, Sharma; GUPTA, Akhil (Ed.). The anthropology of the state: a reader. Malden, 1996. p. 144-162.

ROSE, Nikolas. El gobierno de las democracias liberales avanzadas: del liberalismo al neoliberalismo.Archipiélago, España, n. 29, p. 25-40, 1997.

ROSE, Nikolas; O'MALLEY, Patt; VALVERDE, Mariana. Governmentality. Annual Review of Law and Social Science, Florida, n. 2, p. 83-104, 2006.

SANTIAGO, Rui; CARVALHO, Teresa. Managerialism rhetorics in Portuguese higher education. Minerva, Valladolid, v. 50, n. 4, p. 511-532, dic. 2012.

SUSPITSYNA, Tatiana. Accountability in American education as a rhetoric and a technology of governmentality. Journal of Education Policy, Bielifield, v. 25, n. 5, p. 567-586, 2010.

UACh - Universidad Austral de Chile. Decreto de Rectoría 271/05: orientaciones curriculares de pregrado. Valdivia: UACh, 2005.

UACh - Universidad Austral de Chile. Modelo educacional y enfoque curricular Universidad Austral de Chile. Valdivia: UACh, 2007.

UACh - Universidad Austral de Chile. Perfil piscoeducativo: estudiantes de terapia ocupacional ingreso 2013. Valdivia: UACh, 2013.

UACh - Universidad Austral de Chile. Structuratum renovatio: plan estratégico 2012-2015. Valdivia: UACh, 2012.

WEEDON, Chris. Feminist practice and poststructuralist theory. Oxford: Blackwell, 1987.

YOUDELL, Deborah. Subjectivation and performative politics - Butler thinking Althusser and Foucault: intelligibility, agency and the raced-nationed-religioned subjects of education. British Journal of Sociology of Education, UK, v. 27, n. 4, p. 511-528, sept. 2006.

Recibido en: 18.04.2016

Aprobado en: 17.05.2016

Jimena Carrasco Madariaga es doctora en Psicología Social por la Universidad Autónoma de Barcelona, terapeuta ocupacional, académica del Instituto de Aparato Locomotor y Rehabilitación de la Facultad de Medicina de la Universidad Austral de Chile.

Marcela Apablaza Santis es doctora en Ciencias de la Educación por la Pontificia Universidad Católica de Chile, terapeuta ocupacional, académica del Instituto de Aparato Locomotor y Rehabilitación de la Facultad de Medicina de la Universidad Austral de Chile. 
